\title{
Some Features of the Electrocrystallization of Chromium from Molten Salts
}

\author{
By Tadao Sugaya* and Osamu Watanabe**
}

\begin{abstract}
Chromium crystallizes in various crystal forms, namely, dendrites and platelets, by electrorefining in a bath of fused salt of 1 : $1 \mathrm{NaCl}-\mathrm{KCl}$ containing the trivalent chromium. Crystallographic investigations of these crystals have been carried out. Generally, crystals are bounded with low-index natural facets : i. e., (100), (110), and (111). On the facet (110), however, there are three distinct stripes parallel to either $\langle 001\rangle$ or $\langle 112\rangle$ and $\langle 552\rangle$ directions. Twin growth with the twinning plane $(11 \overline{2})$ is frequently observed, when crystals grow in the $\langle 110\rangle$ direction in the (111) plane or in the $\langle 111\rangle$ direction in the (110) plane. In the molten-salt electro-refining process, both preferential tendency of the orientation and mechanism of the crystal growth should depend strongly on the three factors : i. e., the cathode current-density, the salt-bath temperature, and the electronic structure of metals. In this report, the relative effects of these factors are discussed tentatively.
\end{abstract}

(Received August 22, 1970)

\section{Introduction}

Studies on the crystal growth of chromium single crystals by the molten-salt electrorefining process had not been available, until recently Lenormand and $\mathrm{Graf}^{(1)}$ reported the growth habits of chromium crystals, grown by the electrolysis of melted salts. Their results were related to habits of crystals having a platelike structure with the (111) development, and platelike dendrites showing twin growth developing in the (111) plane. The present study extended their experiments in a comprehensive way to clarify general characteristics of chromium crystals grown by the electrodeposition.

This report deals with some results on the application of the molten-salt electrorefining process for the crystalization of some metals ${ }^{(2)}$ to chromium. In the present investigation, chromium single crystals are precipitated from two different molten electrolytes containing $5 \%$ chromium iodide and $5 \%$ chromium chloride in the $1: 1 \mathrm{NaCl}-\mathrm{KCl}$ solvent, respectively. Chromium crystals, however, grow in the same morphological manner. The growth features and the relative effects of the electrorefining conditions on the crystal growth are described from the phenomenological points of view

\section{Experimental Procedure}

For the electrorefining process $^{(3)}$, impure chromium metal was used as a soluble anode, and the trivalent chromium salts dissolving $5 \% \mathrm{CrCl}_{3}$ or $5 \% \mathrm{CrI}_{3}$ in the $1: 1 \mathrm{NaCl}-\mathrm{KCl}$ solvent as the electrolyte. The electrolysis was carried out under argon atmosphere, and experimental care was taken to exclude air, moisture and oxygenated impurities from the system. Residual impurities in the salt-bath were removed by pre-electrolysis of the solution. After these operations, the electrolysis was performed under various refining conditions of the current-density and the bath-temperature.

* Ohkura Electric CO., Suginami-ku, Tokyo, Japan.

** National Research Institute for Metals, Nakameguro, Tokyo, Japan.

(1) M. Lenormand and R.Graf : Rech. Aerospatiale No. 106, (1965), 21.
From the observation of several hundred chromium crystals grown by the above method, it has been concluded that the morphology of single crystals depend largely on the initial cathode current-density and the bathtemperature. The results on the electrolysis of the molten iodide-bath in the higher temperature region $\left(800^{\circ} \mathrm{C}\right.$ or above) and in the lower current-density region ( $30 \mathrm{amp} / \mathrm{dm}^{2}$ or below) showed that chromium crystallizes generally in two dimensional platelike forms on the cathode face. On the other hand, in the lower temperature region $\left(700^{\circ} \mathrm{C}\right.$. or below) and the higher currentdensity region $\left(100 \mathrm{amp} / \mathrm{dm}^{2}\right.$ or above), chromium crystallizes in the various dendritic forms rather than in the platelike ones. The results obtained from the electrolysis of the molten chloride-bath in the higher temperature region and the lower current-density region showed that chromium crystallizes in the platelike or dendritic forms in the same way as in the iodide-bath operation. As the salt-bath temperature became lower and the current-density higher, the crystals grew in the dendritic forms predominantly. Then, the present experiment showed that there are no remarkable differences between the morphology of chromium crystals grown by the electrolysis of $\mathrm{CrCl}_{3}$ and of $\mathrm{CrI}_{3}$.

Crystallographic aspects of these crystals were examined by back-reflection and transmission Laue Xray techniques and optical goniometric and microscopic methods.

\section{Results and Discussions}

Three distinct dendrites grown independently have been obtained. Two of these dendrites, as shown in Photos. 1 and 2, grow in three intersecting $<100>$ directions. As shown in Photo. 3, the remaining one grows in three $<110\rangle$ directions, forming an angle of $120^{\circ}$ with each other in the basal plane (111) of dendrites. In the early stage of the dendritic growth, it can be divided into two different types: (1) the developing planes are parallel to the (100), as shown in Photo. I;

(2) S. Takeuchi, H. Suzuki and O. Watanabe : Nature, 199(1963), 1283.

(3) O. Watanabe : J. Electrochem. Soc. Japan, 34 (1966), 91. 

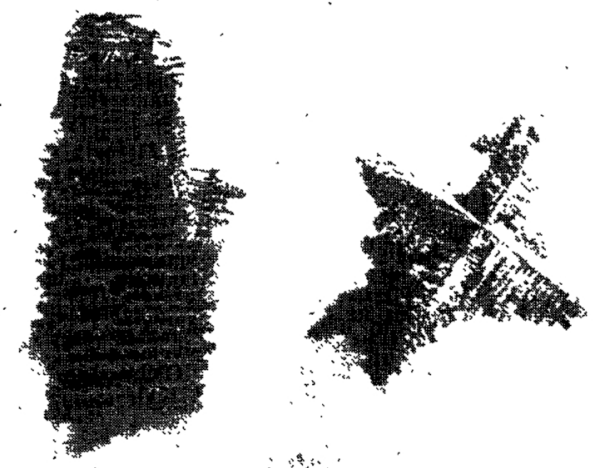

Photo. 1 Dendrites with growth direction $\langle 100\rangle$. $(\times 3)$
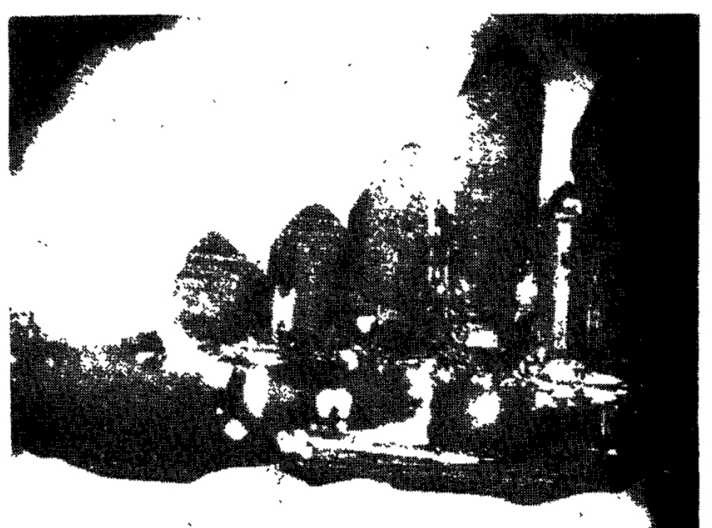

Photo. 2 Dendrites with growth direction $\langle 100\rangle$ and with developing $(100)$ and $(110)$ facets. $(\times 500)$

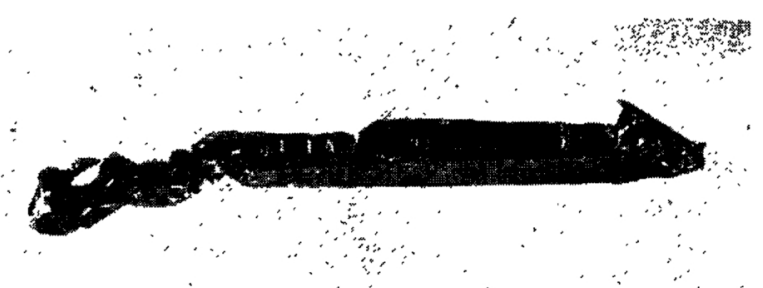

Photo. 3 Dendrite, showing the grooved-sword structure.

and (2) most of them are parallel to the $(110)$ and remainings are parallel to the (100), as shown in Photo. 2. On the other hand, for the dendrites grown as the "groovedsword" shown in Photo. 3, lateral planes and inclined planes consist of $\{111\}$ and $\{110\}$, respectively. The X-ray examination shows that such "grooved-sword" crystals are attended with twin growth, regardless of the sword's length. Piercing through the bottom to the summit of the "grooved-sword", the twinning plane runs parallel to the groove, which is parallel to the one of the three $\langle 110\rangle$ directions in a basal plane (111) and perpendicular to it. Then, the twinning plane is the $(11 \overline{2})$ plane with the growth direction $[1 \overline{1} 0]$, etc. Following the crystal growth in the [110] direction, twin-growth advances. The dendritic feature showing the "grooved-sword" is schematically illustrated in Fig. 1 . The first two kinds of dendrites are grown under the higher current-density operation, and the other under the high current-density $\left(80 \sim 100 \mathrm{amp} / \mathrm{dm}^{2}\right)$ operation.

The platelike forms, shown in Photo. 4 are usually observed. The basal plane facing to the cathode is

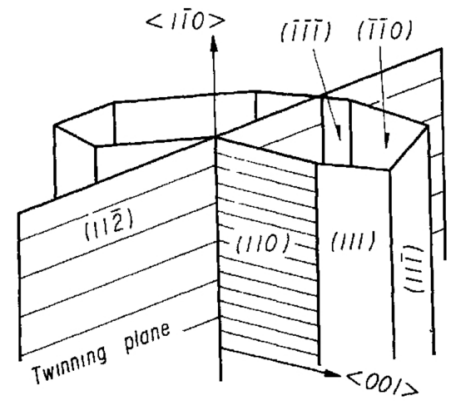

Fig. 1 Diagram to explain the feature of the groovedsword structure.
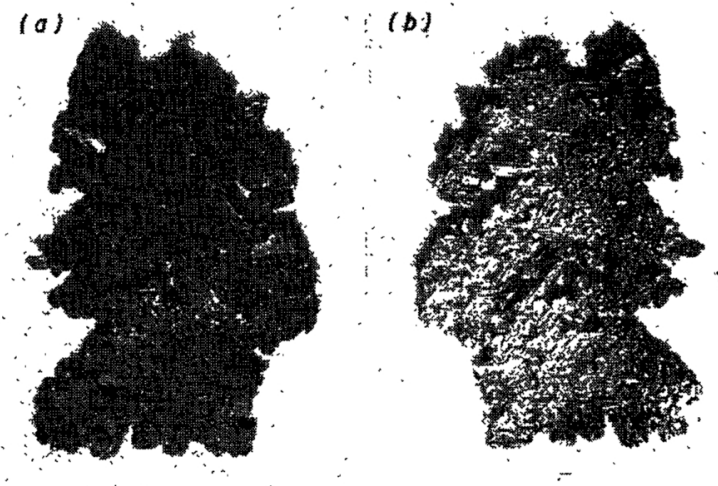

Photo. 4 Crystal grown in a platelike structure. ( $\times 1.5$ ) (a) : the side faced to a cathode b) : the side faced to an anode

parallel to the (111), while the surface facing to the anode side consists of the (111) plane and $\{110\}$ planes, of which the lattter constructs an inclined face with stripes parallel to $\langle 001\rangle$ directions. Such a platelike crystal in the early stage of growth develops in the two-dimensional plane, and observable facets are only of the $\{111\}$ type. The development of the (110) is often followed by the occurrence of the twinning plane. As the crystals grow in the $\langle 110\rangle$ direction in the (111) plane, they tend to fill the basal plane. This type of crystals can be explained in terms of the development of the "grooved-sword" structure. These crystals are obtained under the operation with higher bath-temperature (above $800^{\circ} \mathrm{C}$ ) and the lower currentdensity (below $30 \mathrm{amp} / \mathrm{dm}^{2}$ ).

As shown in Photo. 5, the crystals grow frequently as a "coral-reef" forms, showing the twin growth. The basal plane is parallel to the (110) with stripes (Photo. 6) parallel to the $[\overline{5} \overline{5} \overline{2}]$ and the [112]. The growth directions in the basal plane are parallel to either $<111\rangle$ or $\langle 11 \overline{2}\rangle$. For the growth direction $\langle 111\rangle$, the twinning plane $(11 \overline{2})$ is perpendicular to the basal plane $(1 \overline{1} 0)$ and to the (111) plane. Crystals with the "coral-reef" growth develop along the [41 $\overline{5}$ ] direction in a $(2 \overline{3} 1)$ plane from the twinning plane $(11 \overline{2})$ as a boundary plane, showing the step-on-step surface structure. In other words, the plates grown in the [112] direction lying in the $(1 \overline{1} 0)$ plane are piled up with a constant up-grade to the $[1 \overline{1} 0]$, thus forming crystals of the step-on-step structure. An up-grade is lying in the $(2 \overline{3} 1)$ plane, and a piled-up direction is [415]. The step-on-step surface is parallel to the basal plane, and 


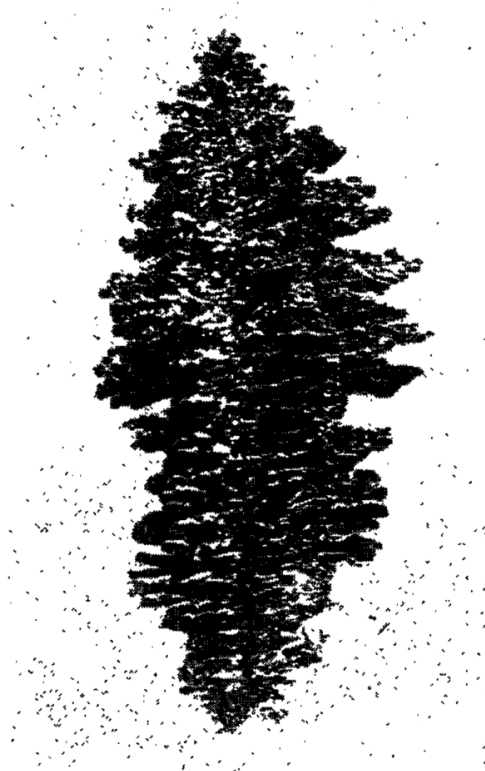

Photo. 5 Development of a coral-reef structure with twin growth. $(\times 2)$

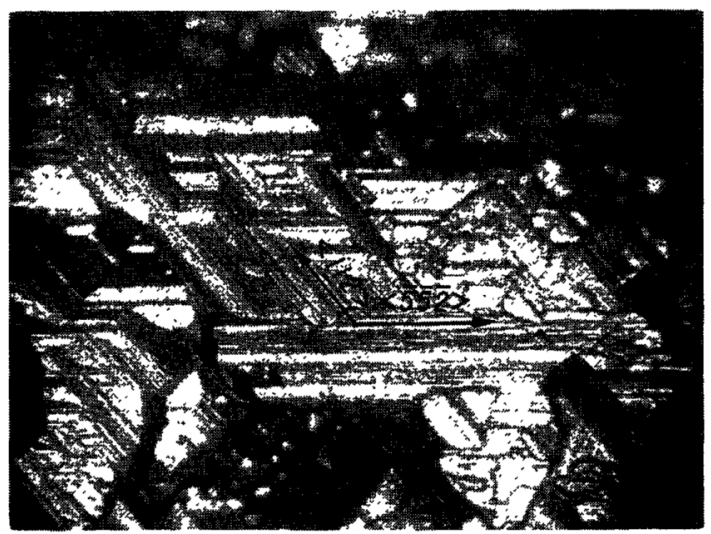

Photo. 6 Enlargement of a basal plane of the coral-reef structure. Stripes are parallel to either $\langle 112\rangle$ or $\langle\overline{5} \overline{2} \overline{2}\rangle$. $(\times 250)$

the same stripes are found on this surfaces as on the basal plane. In this sense, the development of the $(2 \overline{3} 1)$ is incomplete. The "coral-reef" structure is diagrammatically shown in Fig. 2.

The general features of the octahedral or polyhedral growth are shown in Photos. 7 and 8, showing two distinct growth characteristics; one (Photo. 7) is reduced to the system developed from the dendrite shown in Photo. 1, and the other to that from the plate structure shown in Photo. 4. In either case, the crystals grow in three intersecting $\langle 100\rangle$ directions. A tentative explanation of the octahedral and/or polyhedral growth features may be as follows: The edges of crystals grow in $\langle 100\rangle$ directions and then accelerate the development of (111) family planes with further growth in a second $\langle 110\rangle$ direction. Moreover, the (110) and (100) planes, cutting out summits and edges of the octahedron respectively, are developed by the reentrance $<100\rangle$ growth. As shown in Photos. 7 and 8 , the octahedron (or polyhedron) shows the growth of a new octahedron (or polyhedron) at an apex, constructing a dendritic "cup-on-cup" structure. In Fig. 3, the

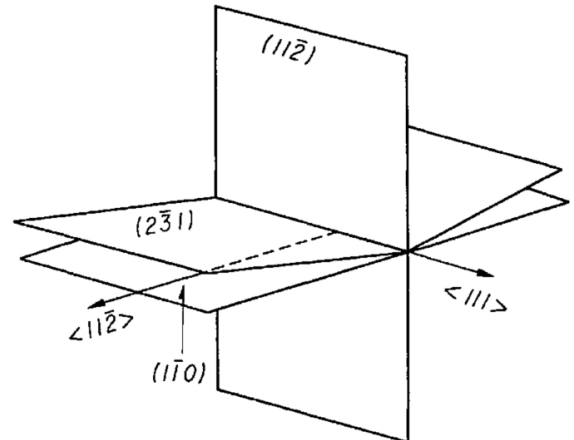

Fig. 2 A schematic diagram of the coral-reef structure with a twinning plane.

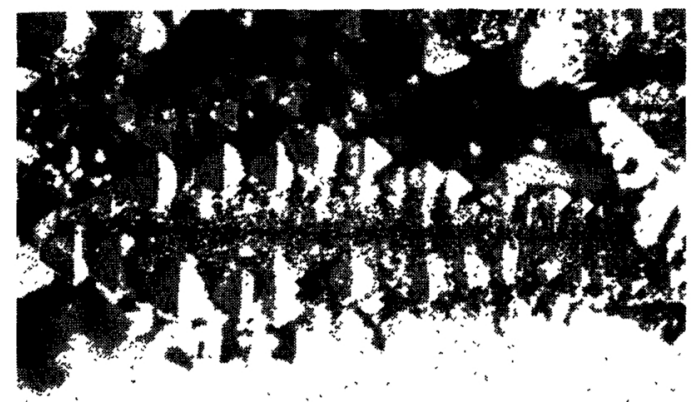

Photo. 7 Dendritic octahedral growth. $(\times 25 \times 4 / 5)$

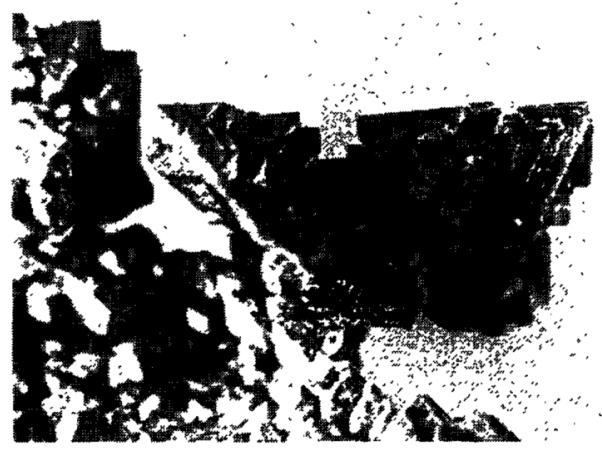

Photo. 8 Development of the cup-on-cup structure, showing octahedral growth. $(\times 8)$

octahedral growth from the platelike structure is predicted schematically. These crystals are grown under the intermediate temperature operation with currentdensities of nearly $50 \mathrm{amp} / \mathrm{dm}^{2}$.

Photo. 9 (a) shows that the developing planes are dominantly three intersecting $\{100\}$ facets, which constitute a multi-layered structure. In addition to $\{100\}$ facets, $\{111\}$ and $\{110\}$ are rarely found. It is assured that at a certain stage the crystal grows in the [111] direction with developing $\{100\}$. In the early growing stage, there is a pyramidal hillock bounded by $\{111\}$ or $\{110\}$ on the (100) facet, as shown in Photo. $9(\mathrm{~b})$. A growth pattern on the $(100)$ shows that an edge nucleation of the pyramidal hillock occurs from edges parallel to the three intersecting $\langle 100\rangle$ directions. The development of the (111) facet layers is followed by $<110\rangle$ growth in $\{111\}$ planes. On the crystals with a "hopper-opening" structure, which are shown in Photo. 10, the preferential developing planes are $\{111\}$ 


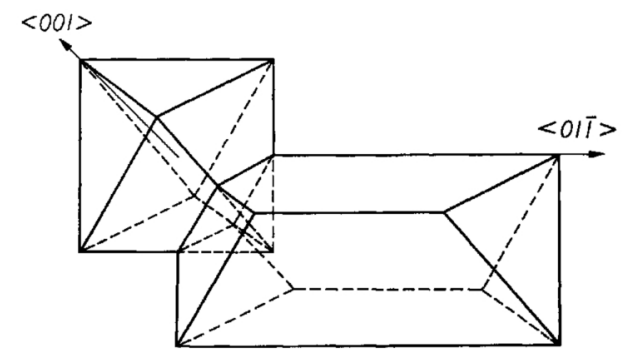

Fig. 3 A schematic diagram of the octahedral growth (Photo. 8). Planes are $\{111\}$. structure, which include the distances between atoms on the axis $\langle 100\rangle,\langle 110\rangle$, and $\langle 111\rangle$, and the number of atoms per unit area of the planes $(100),(110)$, and (111). They are listed in Table 1. Using Table 1, the growth directions, may be defined as follows : (1) When the direction $\langle 111\rangle$, the closest packed direction in the $b c c$ structure, is the preferential growth axis, it gives rise to the secondary growth in three intersecting directions $\langle 100\rangle$ which are the next close packed directions, (2) when the next close packed

(a)
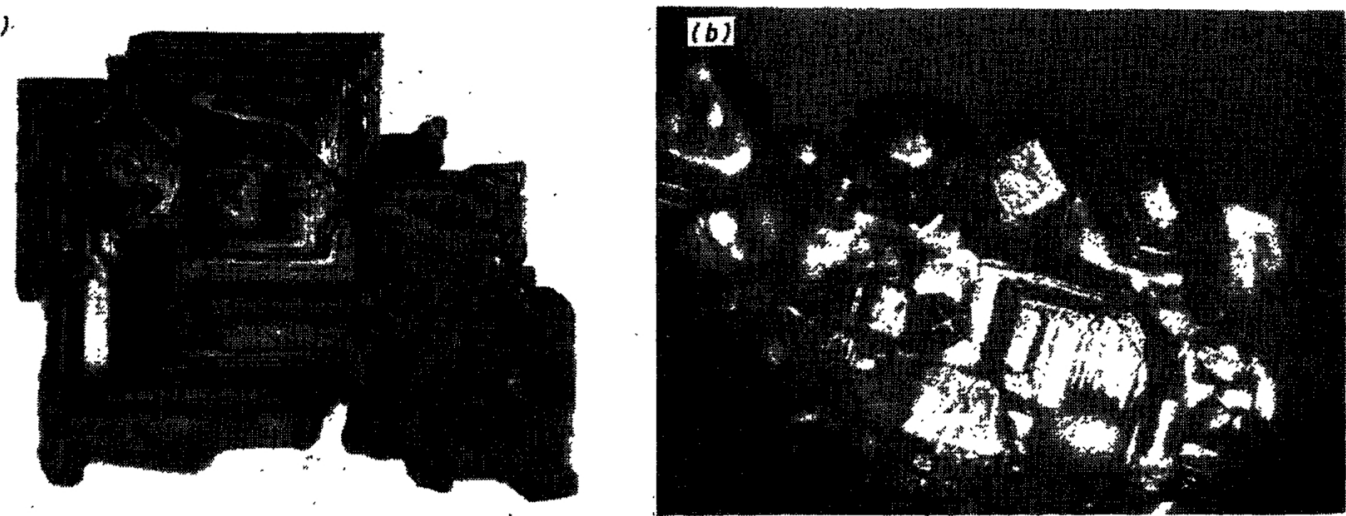

Photo. 9 (a). Crystal with the step-on-step structure. $(\times 30)$

(b) Pyramidial hillocks grown on the (100) facet, one of them is bounded by the (110) facet, and the other by the (111) facet. $(\times 600)$

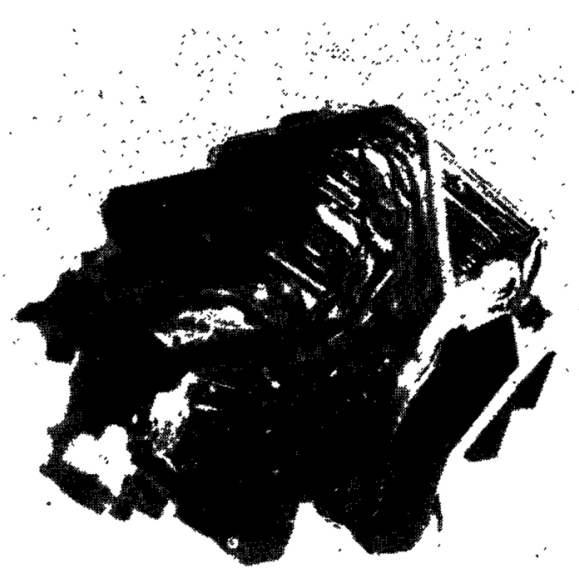

Photo. 10 A hopper prismatic column showing the stepped basal and prism surface. $(\times 35)$

and $\{100\}$. The other developing planes are $\{110\}$, which have stripes parallel to $\langle 001\rangle$. For these crystals, the growth directions may be explained by their growth to $\langle 110\rangle$ and $\langle 100\rangle$ with the principal [111] axis growth. Figure 4 shows diagramatical features of the "hopper-opening" structure in Photo. 10. Frequently, fully advanced (111). facets, are found. The features of filling-up of $\{111\}$ are shown in Photo. 11, showing the "step-on-step" structure and the edge nucleation.

In order to investigate the relationship between the growth directions, a hard sphere model can be assumed for a body centered cubic structure. We can easily tabulate some crystallographic characteristics of the $b c c$

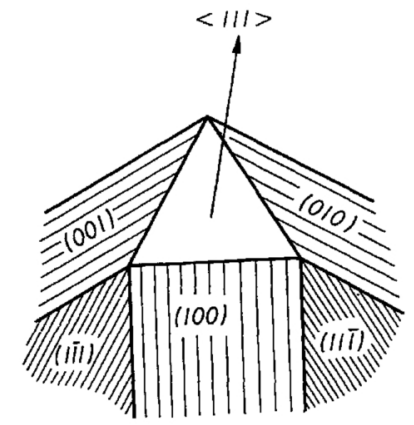

Fig. 4 A schematic diagram to explain the hopper development shown in Photo. 10.

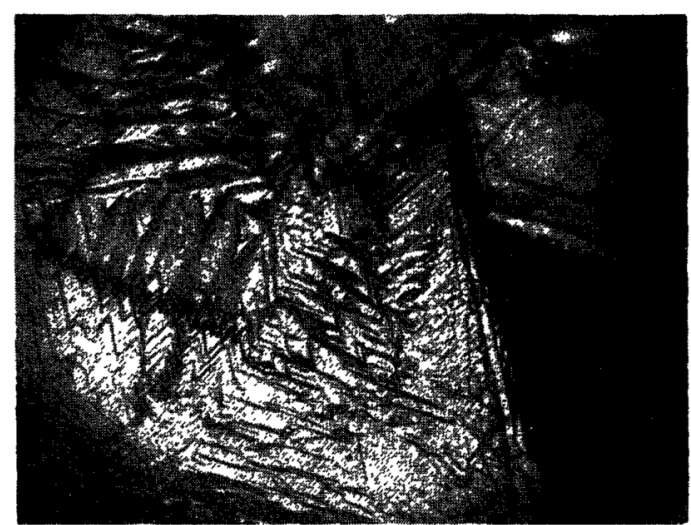

Photo. 11 Filling-up of the (111) facet, showing the step-on-step structure with constant thickness and edge nucleation.

direction $\langle 100\rangle$ is the preferential growth axis, it gives rise to the secondary growth in the same direction or the third close packed one $\langle 110\rangle$, and (3) when the third close packed direction $\langle 110\rangle$ is 
Table 1 Crystallographic characteristics of the body-centered cubic structure.

\begin{tabular}{c|c|c|c|c}
\hline $\begin{array}{c}\text { Develop- } \\
\text { ing plane }\end{array}$ & $\begin{array}{c}\text { Growth } \\
\text { axis }\end{array}$ & $\begin{array}{c}\text { Number of atoms } \\
\text { per unit area } \\
\left(\times a^{-2}\right)\end{array}$ & $\begin{array}{c}\text { Layer } \\
\text { spacing } \\
(\times a)\end{array}$ & $\begin{array}{c}\text { Distance between } \\
\text { atoms on the } \\
\text { growth axis }(\times a)\end{array}$ \\
\hline 100 & 100 & 1 & $1 / 2$ & 1 \\
110 & 110 & $\sqrt{2}$ & $1 / \sqrt{2}$ & $\sqrt{2}$ \\
111 & 111 & $1 / \sqrt{3}$ & $1 / 2 \sqrt{3}$ & $\sqrt{3} / 2$ \\
112 & 112 & $2 / \sqrt{6}$ & $1 / \sqrt{6}$ & $\sqrt{6}$ \\
\hline
\end{tabular}

where $a$ is a lattice constant change conspicuously, since a crystal deposited on the electrode develops and maintains a definite form : the morphology of crystals does not show any remarkable change. Thus, these facts suggest that the initial conditions of the electrorefining process, i. e., the bathtemperature and the cathode current-density, are maintained without a marked variation during the crystal growth. Therefore, the morphology of crystals on the electrode seems to depend largely on the initial conditions of the electrorefining process rather than the

Table 2 Growth features of chromium crystals.

\begin{tabular}{c|c|c|c|c|c|c}
\hline $\begin{array}{c}\text { Preferential } \\
\text { growth axis }\end{array}$ & $\begin{array}{c}\text { Secondary } \\
\text { growth } \\
\text { directions }\end{array}$ & $\begin{array}{c}\text { Preferential } \\
\text { developing } \\
\text { planes }\end{array}$ & $\begin{array}{c}\text { Existence } \\
\text { of twin } \\
\text { growth }\end{array}$ & $\begin{array}{c}\text { Forms } \\
\text { or } \\
\text { Structures }\end{array}$ & $\begin{array}{c}\text { Electrorefining conditions } \\
\text { Current-density } \\
\left(\text { amp/dm }{ }^{2}\right)\end{array}$ & $\begin{array}{c}\text { Bath-temperature } \\
\left({ }^{\circ} \mathrm{C}\right)\end{array}$ \\
\hline 100 & 100 & 100 & none & dendrite & $100<$ & $<700$ \\
\cline { 2 - 7 } & 110 & 111 & none & $\begin{array}{c}\text { platelike, } \\
\text { (cup-on-cup) }\end{array}$ & $<00<$ \\
\hline 110 & 100 & 111 & exist & $\begin{array}{c}\text { platelike dendrite, } \\
\text { (grooved-sword) }\end{array}$ & $<30$ & $800<$ \\
\hline \multirow{2}{*}{111} & 110 & 110 & none & $\begin{array}{c}\text { step-on-step, } \\
\text { cup-on-cup }\end{array}$ & intermediate \\
\hline \hline
\end{tabular}

the preferential growth axis, it gives rise to the secondary growth in the same direction and/or the forth close packed direction $\langle 112\rangle$. In the absence of twin growth, we arrive at the well-known conclusions. The occurrence of the twin growth makes a conspicuous change in the growth feature. The twinning plane is always the one of $\{112\}$ planes. When the twinning plane is parallel to growth directions $\langle 110\rangle$ in the (111) plane, it leads to the dendritic crystals with the "grooved-sword" structure, accelerating their growth in the $\langle 001\rangle$ and other $\langle 110\rangle$ directions. When the twin growth is parallel to the $\langle 111\rangle$ direction in the (110) plane, it leads to the platelike crystals with the "coral-reef" structure, accelerating their growth in $<112>$ directions. The growth features of chromium crystals are tabulated in Table 2 . In any growth stage, our experiment shows that the development of $\{110\}$ planes is incomplete. It has always three distinct stripes running parallel to either $\langle 001\rangle$ or $\langle 112\rangle$ and $<552>$ directions. Phenomenologically, if we can roughly regard the generation of striped (110) facet as a transition stage which leads to the nucleation of the other planes such as $\{100\}$ and $\{111\}$, the stripes may be characterised by the transition stage in this sense. However, the development of the incomplete (110) facet is in contrast to the usual result that since the atomic planes of closest-packing have the lowest surface energy, these planes predominate in the surface facets of equilibrium crystals. It is comprehensible that the morphology of crystals is usually determined by the rate of crystal growth rather than by the equilibrium shape. Our results, however, suggest that the rate of crystal growth, during the electrorefining process, does not rate of crystal growth. Now, in the case of investigating the preferential tendency of the crystal growth, it is essential to take account of the atomic and the electronic structure of a metal. Although there are a number of theories on the electronic structure of metals, the concept of Wigner and Seitz ${ }^{(4)}$ touches upon the theoretical essence. According to them, the WignerSeitz cell is chosen by taking the polyhedron whose plane faces bisect orthogonally the lines joining an atom with its nearest neighbors. In the case of the $b c c$ structure, this polyhedron is composed by two kinds of planes, i.e., $\{100\}$ and $\{111\}$. Since it is possible that the potential between atoms is minimum on the planes of the polyhedron, the crystal bounded by low-index facets can be in equilibrium shape. This form reflects the characteristic symmetry of the unit cell. According to the electronic structure of chromium ${ }^{(5)}$, the electronic transport properties, showing an appreciable anisotropy about $\langle 100\rangle$ axis, reflect the geometrical form of the Fermi surface of chromium. This fact intuitively suggests to us that the $<111>$ direction can be the most favourable growth direction. The crystal lattice potential has the maximum at the corner corresponding to $(\pi / a, \pi / a, \pi / a)$ in the Brillouin zone which is bounded by the $\{110\}$ planes, where $a$ is a lattice constant. It is a possible assumption that the (111) facet can be the most stable facet reflecting the crystal potential. Since the work function of an atom for each low-index facets has not been known, we cannot

(4) J.M. Ziman : Principles of the Theory of Solids, Cambridge University Press, London, (1964), p. 85.

(5) L. M. Faricov and M. J. Zuckermann : Phys. Rev., 160 (1967), 372. 
make more advanced discussion about the preferential tendency of the crystal growth, which is phenomenologically dependent on the cathode current-density and the bath-temperature. It is very difficult, in the present study, to describe how the crystallization by the electrorefining process is characterized by the correlations among the current-density, the bath-temperature, and the electronic structure of the metal, in terms of thermodynamics. However, it will be future problems to relate the electronic structure to the crystal growth mechanism.

\section{Conclusions}

Chromium crystallizes in single crystal form, by the molten-salt electrorefining process using the trivalent chromium in the $1: 1 \mathrm{NaCl}-\mathrm{KCl}$ electrolyte solvent. With respect to the crystallization, it may be concluded that the variation of the salt-bath temperature and the cathode current-density have distinct effects on the crystal growth features. When the electrolysis is operated in the low temperature region (below $700^{\circ} \mathrm{C}$ ) and the high current-density region (above $100 \mathrm{amp} / \mathrm{dm}^{2}$ ), chromium crystallizes in a dendritic manner; in the higher temperature region (above $800^{\circ} \mathrm{C}$ ) and the lower current-density region (below $30 \mathrm{amp} / \mathrm{dm}^{2}$ ), in a platelike manner ; and in the intermediate conditions, in a mixed manner of the above two.

The crystal develops and maintains a simple definite form during the electrorefining process. The bounding planes and the growth directions are characterized by low-index numbers, i.e., $\{100\}$ and $\{111\}$, and $<100>$, $\langle 110\rangle$, and $\langle 111\rangle$. In any crystallization, the (110) facet develops incompletely. It has always three distinct stripes parallel to either $\langle 001\rangle$ or $<552\rangle$ and $<112\rangle$, depending on the growth directions : i.e., the latter take $\langle 111\rangle$ directions in the (110), and the former can be found generally. The twinning plane is $\{112\}$. There are two kinds of twin growth ; one is parallel to the three distinct $\langle 110\rangle$ in the (111), and the other to the two distinct $\langle 111\rangle$ in the (110).

In the molten-salt electrorefining process, both preferential tendency of the orientation and mechanism of the crystal growth should be strongly dependent on the three factors, i.e., the cathode current-density, the bathtemperature, and the electronic structure of chromium. In the present investigation, we cannot discuss the mutual relation among these factors on the crystal growth mechanism, because the experimental results are always complicated by the difficult problems owing to the control of the cathode current-density. It is recommended that in order to solve these problems further investigations on the electrodeposition of metals should be carried out systematically.

\section{Acknowledgments}

This work was supported in part by the Scientific Research Funds of the Ministry of Education. In addition, one of the authors (T.S.) expresses his appreciation to the Ohkura Electric Co. 